\title{
Tom Bevel, Ross Gardner: Bloodstain Pattern Analysis, 3rd Edition
}

\author{
Dean A. Wideman
}

Published online: 29 July 2008

(C) Humana Press 2008

Bloodstain pattern analysis (BPA) can play an important role in understanding the actions and events that occurred during a criminal incident. Mr. Bevel and Mr. Gardner have expanded on the earlier editions of this book and although bloodstain pattern analysis can be a complicated and highly technical subject, this book, although comprehensive, is written in a manner that is easy to understand.

This book will be useful and beneficial to a variety of forensic practitioners, law enforcement officials, attorneys, and university students who want to learn more about the theory, principles, and application of bloodstain pattern analysis. The book includes chapters on the history of BPA, general bloodstain pattern terminology, classifications of bloodstains, methodology of BPA, blood dynamics, determining motion and directionality, determining point of convergence and point of origin, impact spatter stains, presumptive testing and enhancement of blood, documenting bloodstains, introduction to crime scene reconstruction, presenting bloodstain pattern evidence, etc. The book also contains a variety of useful diagrams, illustrations, and photographs to provide the reader with visual references for better understanding of the theory and concepts being discussed.

This book answers a lot of questions regarding bloodstain pattern analysis and its application to understanding the actions and events that took place during crimes where bloodshed occurred. This book also helps the non-scientist reader understand the principles and complexities of bloodstain pattern analysis and enables him to gain an appreciation of the science and developments within this field.

Mr. Bevel and Mr. Gardner have published a valuable reference book that should be included in the library of all crime laboratories and police departments and have provided an essential text for those students who are currently studying bloodstain pattern analysis.
D. A. Wideman ( $\square)$

NucleoGenix LLC, San Antonio, TX, USA

e-mail: dwideman@forensic-profiler.com 